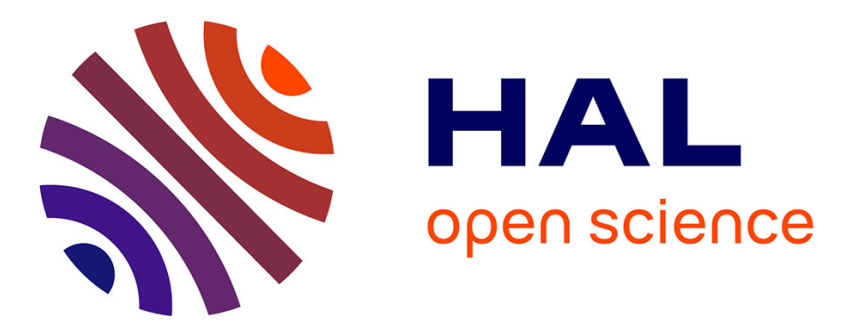

\title{
Muscle alterations induced by electrostimulation are lower at short quadriceps femoris length
}

Alexandre Fouré, Augustin C. Ogier, Maxime Guye, Julien Gondin, David Bendahan

\section{- To cite this version:}

Alexandre Fouré, Augustin C. Ogier, Maxime Guye, Julien Gondin, David Bendahan. Muscle alterations induced by electrostimulation are lower at short quadriceps femoris length. European Journal of Applied Physiology, 2019, 120, pp.325 - 335. 10.1007/s00421-019-04277-5 . hal-02426083

\section{HAL Id: hal-02426083 \\ https://hal.science/hal-02426083}

Submitted on 14 Feb 2021

HAL is a multi-disciplinary open access archive for the deposit and dissemination of scientific research documents, whether they are published or not. The documents may come from teaching and research institutions in France or abroad, or from public or private research centers.
L'archive ouverte pluridisciplinaire HAL, est destinée au dépôt et à la diffusion de documents scientifiques de niveau recherche, publiés ou non, émanant des établissements d'enseignement et de recherche français ou étrangers, des laboratoires publics ou privés. 


\title{
Muscle alterations induced by electrostimulation are lower at short quadriceps femoris length
}

\author{
Alexandre Fouré ${ }^{1,2,3} \cdot$ Augustin C. Ogier ${ }^{4} \cdot$ Maxime Guye ${ }^{1,2} \cdot$ Julien Gondin ${ }^{1,5} \cdot$ David Bendahan $^{1}$
}

\begin{abstract}
Purpose This study aimed at determining through MRI investigations, force and soreness assessments whether the modulation of muscle length is a relevant strategy for minimising neuromuscular electrical stimulation (NMES)-induced muscle damage in young healthy participants.

Methods Comparison of 2 NMES sessions (40 isometric electrically-evoked contractions of the knee extensors) was randomly performed on 1 knee flexed at $50^{\circ}$ (short muscle length) and the contralateral at $100^{\circ}$ (long muscle length) in a single group of healthy participants. Indirect markers of muscle damage including changes in maximal voluntary isometric contraction (MVC) force, muscle volume and transverse relaxation time $\left(\mathrm{T}_{2}\right)$ were measured before, 2 days (D2), 4 days (D4) and 7 days (D7) after the NMES sessions in each limb of the ten participants.

Results Although stimulation intensity was similar during the NMES session on both limbs, significantly lower force production was recorded at long muscle length (peak at $30 \pm 5 \% \mathrm{MVC}$ force) as compared to short muscle length (peak at $61 \pm 12 \%$ MVC force). In the following days, MVC force at long muscle length was decreased from D2 to D7, whereas no significant change occurred at short muscle length. Increases in muscle volume and $T_{2}$ were found at each time point in stimulated muscles at long muscle length, whereas no change was found at short muscle length.

Conclusion For the same stimulation intensity, NMES-induced isometric contractions generate higher knee extension force output and result in lower muscle tissues alterations that could be related to a lower intramuscular shear strain when exercise is performed at short muscle length.
\end{abstract}

Keywords MRI $\cdot$ Muscle damage $\cdot$ Acute exercise $\cdot$ Neuromuscular electrical stimulation $\cdot$ Muscle strength $\cdot$ Thigh

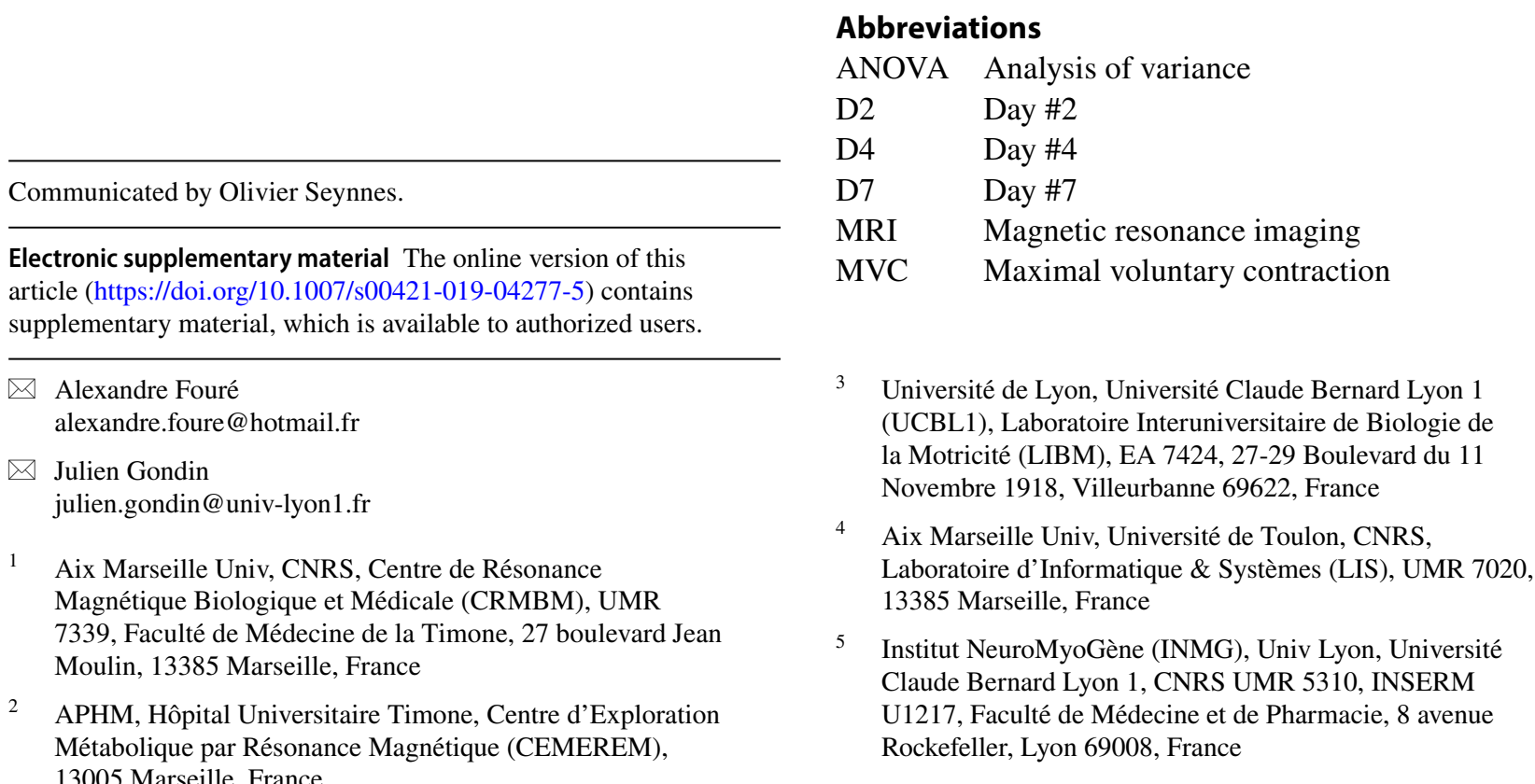




$\begin{array}{ll}\text { NMES } & \text { Neuromuscular electrical stimulation } \\ \text { POST } & \text { Immediately after the NMES session } \\ \text { PRE } & \text { Before the NMES session } \\ \text { RF } & \text { rectus femoris } \\ \text { SAR } & \text { sartorius } \\ T_{2} & \text { Transverse relaxation time } \\ \text { VAS } & \text { Visual analog scale } \\ \text { VI } & \text { vastus intermedius } \\ \text { VL } & \text { vastus lateralis } \\ \text { VM } & \text { vastus medialis }\end{array}$

\section{Introduction}

Repetition of unaccustomed maximal voluntary eccentric or isometric contractions can lead to muscle damage (Armstrong 1984; Crameri et al. 2007; Nosaka et al. 2011). Structural alterations of muscle organization have been reported within days following the damaging exercise (Crameri et al. 2007; Mackey et al. 2011). These changes were accompanied by decreased maximal voluntary isometric contraction (MVC) force (Clarkson et al. 1992; Fouré et al. 2015b) and range of motion (Clarkson et al. 1992; Nosaka and Clarkson 1996), muscle swelling (Howell et al. 1993; Fouré et al. 2014), increased muscle stiffness (Lacourpaille et al. 2014, 2017; Xu et al. 2018), plasmatic muscle protein concentration (Clarkson et al. 1992; Nosaka and Clarkson 1996; Fouré et al. 2014) and delayed onset muscle soreness (Howell et al. 1993).

Interestingly, it has been suggested that the magnitude of muscle damage induced by unaccustomed exercises could be modulated by various factors. For instance, the amount of muscle damage has been found to be higher in upper limb muscles as compared to the lower limb ones (Jamurtas et al. 2005; Saka et al. 2009). Moreover, it has been demonstrated that muscle damages were larger during eccentric exercise performed at a long muscle length and for a large range of motion as compared to those resulting from eccentric exercise performed at short muscle length and small range of motion (Nosaka et al. 2005). Similarly, eccentric exercise involving high force generation and a high number of repetitions can cause large muscle fibre structural alterations (Lieber and Friden 1993; Paulsen et al. 2012). Using real-time ultrasound imaging, it has been reported that the amount of fascicle length changes during maximal voluntary eccentric contractions and then the level of negative work imposed by the exercise is highly involved in the subsequent MVC force loss (Guilhem et al. 2016; Doguet et al. 2019). Muscle damage has also been reported as a result of intense voluntary isometric contractions ( $>60 \% \mathrm{MVC}$ ) performed at long muscle length (Allen et al. 2018) even if muscle force and length change were lower than what could be generated during maximal voluntary eccentric contractions.
Deleterious effects of isometric contractions have also been reported as a result of neuromuscular electrical stimulation (NMES) (Fouré et al. 2014, 2015a). To our knowledge, these changes have only been reported for contractions performed at long muscle length and at relatively low force levels ( 30\% MVC) (Aldayel et al. 2010; Fouré et al. 2015a) so that the damaging effects of isometric contractions performed at short muscle length have not been assessed. On the contrary to what occurs during voluntary contractions, the motor units' recruitment during NMES is synchronous, spatially fixed and involves fast and slow motor units at the same time (Maffiuletti 2010). Such an activation of fast muscle fibres even at relatively low levels of evoked force (Gregory and Bickel 2005) can induce early fatigue and generate muscle damage and extracellular matrix deadhesion (Mackey et al. 2008, 2011). Based on the "popping sarcomere hypothesis", fast muscle fibres have a shorter optimum length (Brockett et al. 2002; Allen et al. 2018) and are more prone to alterations (Morgan and Proske 2004). One can hypothesize that fibres' alterations could be alleviated during NMES-induced isometric contractions performed at short muscle length, considering a lower strain (i.e., the relative fascicle length change) reported to be a key factor in the force loss after maximal eccentric plantarflexions (Guilhem et al. 2016).

The aim of the present study was to assess the effects of muscle length on the occurrence and extent of NMESinduced muscle damage on the basis of multimodal MRI investigations, force and soreness assessments conducted over a 7-day post-exercise period.

\section{Methods}

Ten healthy participants $(27 \pm 4$ years, $173 \pm 10 \mathrm{~cm}$, $69 \pm 10 \mathrm{~kg}$, four women) volunteered to participate in this study. None of them were engaged in any training or exercise programs. Participants were instructed to avoid any intensive and non-familiar physical activities throughout the duration of the protocol. They were fully informed about the nature and aims of the study and gave their informed written consent to participate. The study was approved by the Local Human Research Ethics Committee (Sud Mediterranée V, \#2012-04 A00449-34) and conducted in conformity with the Declaration of Helsinki. Participants were asked to keep their diet habits and limit their alcohol consumption throughout the study period. They were instructed to avoid consuming caffeine and smoking before the experiments. Consumption of medication was also prohibited during the experimental protocol. All testing sessions were performed at the same time of day. The experiment included four sessions aiming at assessing muscle function and tissue integrity before (PRE), immediately after the NMES session 


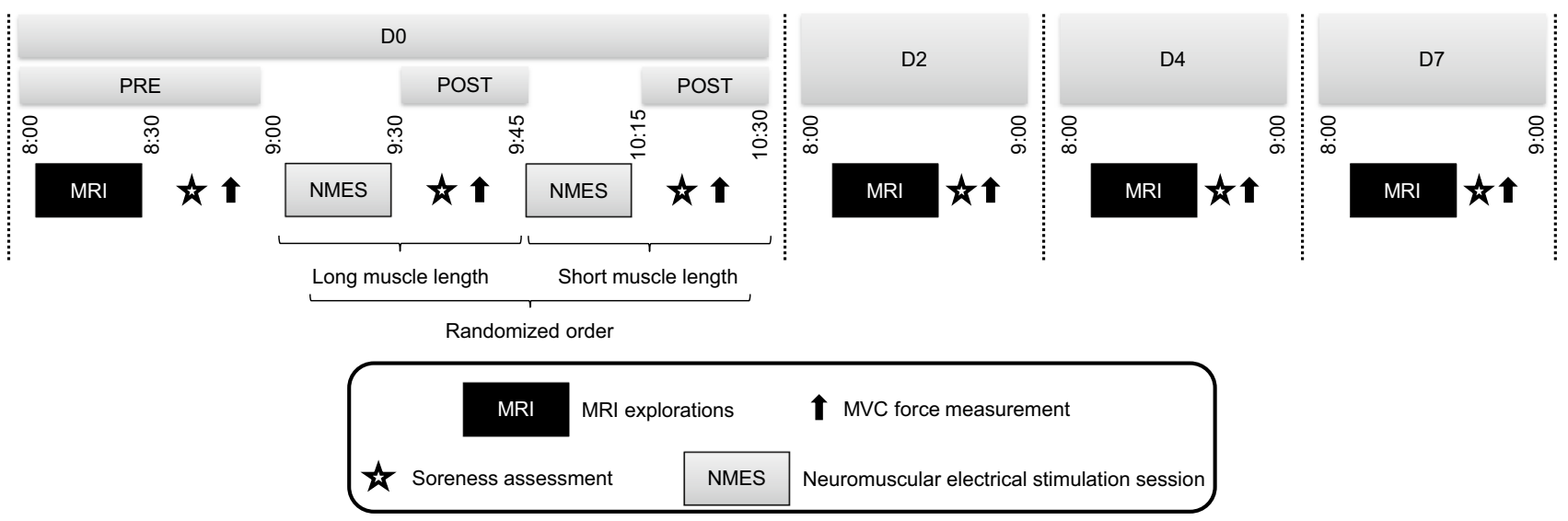

Fig. 1 Schematic representation of the experimental design

(POST) and in the following days (two [D2], four [D4] and seven [D7]-Fig. 1).

Participants were seated on a chair (Multi-Form', La Roque d'Anthéron, France) customized with a force sensor. Adjustable belts secured hip and ankle joints to hold the hip at $\sim 90^{\circ}\left(0^{\circ}\right.$ corresponding to the joint fully extended). Similar NMES sessions were randomly performed either with the knee flexed at $100^{\circ}$ corresponding to a long muscle-tendon length (hereafter referred to as long muscle length) and with the contralateral knee flexed at $50^{\circ}$ corresponding to a short muscle-tendon length (hereafter referred to as short muscle length). Link between knee joint angle and muscle fascicle length was performed on the basis of ultrasound imaging measurements on the vastus lateralis during eccentric contractions with a mean fascicle length of $85 \mathrm{~mm}$ and $100 \mathrm{~mm}$ at a knee flexion angle of $40^{\circ}$ and $100^{\circ}$, respectively (Doguet et al. 2019). Considering that VL muscle slack length was assessed for a knee flexion angle of $24^{\circ}$ [range $16^{\circ}-30^{\circ}$ ] (Stubbs et al. 2018), we assume that the two testing positions were beyond the VL slack length (Stubbs et al. 2018; $\mathrm{Xu}$ et al. 2018). The two different NMES conditions of the present study correspond to knee joint flexion angles that can be used in a clinical context to minimise muscle atrophy in patients. Knee extensor muscles were stimulated using three electrodes placed over the thigh, a $5 \times 10 \mathrm{~cm}$ rectangular electrode on the proximal part of the thigh (i.e., placed $\sim 5 \mathrm{~cm}$ below the inguinal ligament) and two $5 \times 5 \mathrm{~cm}$ square electrodes on the vastus lateralis $(\mathrm{VL})$ and vastus medialis (VM) muscle bellies. Biphasic symmetric rectangular pulses were delivered at a frequency of $100 \mathrm{~Hz}$ with a pulse duration of $400 \mu \mathrm{s}$ (40 contractions, $5 \mathrm{~s}$ on and $35 \mathrm{~s}$ off throughout the NMES session) using a portable battery-powered stimulator (Compex ${ }^{\circledR}$ Performance, DjoGlobal, France). Stimulation intensity was gradually increased during the 40 contractions to reach the highest tolerated (considering the pain threshold) level of electrically-evoked force for each participant as previously described (Fouré et al. 2014, 2015a). Stimulation intensities and electrically-evoked force outputs were recorded every five contractions during the entire series of each NMES session. Evoked force was normalized to MVC performed at the corresponding muscle length.

Each participant was seated on a chair with the knee flexed (i.e., at $100^{\circ}$ for one knee and $50^{\circ}$ for the other) and performed a 5-min warm-up including a set of sub-maximal knee extensions under isometric conditions. MVC was assessed on each side in the same position used for the NMES session. Participants were instructed to perform three unilateral MVC. The MVC trials were separated by a resting period of at least $30 \mathrm{~s}$. MVC force was considered as the highest value recorded among the three trials.

The level of muscle soreness was assessed by the same experimenter (A.F.) using circular palpation (Lau et al. 2015) on the VL and the VM muscle bellies at rest (i.e., before MVC trials) and using a visual analog scale (VAS) with a $100 \mathrm{~mm}$ horizontal line with "no pain" on one end $(0 \mathrm{~mm})$ and "extremely painful" on the other $(100 \mathrm{~mm})$. An additional measure was performed immediately after MVC trials. Soreness was quantified as the mean value obtained from the three measures.

Participants were positioned supine with the limb of interest centred in a 1.5-T super-conducting magnet (MAGNETOM Avanto, Siemens AG, Healthcare Sector, Erlangen, Germany). A flexible surface six-channel coil (Siemens AG, Healthcare Sector, Erlangen, Germany) was placed around the thigh muscles. Muscle volume was determined from high-resolution $T_{1}$-weighted images $(20$ slices, field of view $=220 \mathrm{~mm} \times 220 \mathrm{~mm}$; matrix $=576 \times 576$; relaxation time $=549 \mathrm{~ms}$; echo time $=13 \mathrm{~ms}$; number of repetitions $=1$; slice thickness $=6 \mathrm{~mm}$; gap between slices $=6 \mathrm{~mm}$, acquisition time $=5 \mathrm{~min} 18 \mathrm{~s}$ ). $\mathrm{T}_{2}$-weighted 
images were acquired with a segmented (15 segments) echo planar imaging sequence with time echos $=15,25,35,45$ and $55 \mathrm{~ms}$. Other acquisition parameters were as follows: field of view $=220 \mathrm{~mm} \times 220 \mathrm{~mm}$; matrix $=192 \times 192$; repetition time $=4800 \mathrm{~ms} ; \mathrm{N}_{\mathrm{EX}}=1$; number of slices $=20$; slice thickness $=6 \mathrm{~mm}$; gap between slices $=6 \mathrm{~mm}$, fat saturation; acquisition time $=5 \mathrm{~min} 10 \mathrm{~s}$. The most distal slice was always acquired at approximately $20 \mathrm{~mm}$ (i.e., $5 \%$ of the thigh length measured for each participant) above the proximal border of the patella.

Regions of interest (i.e., thigh muscles including the VL, $\mathrm{VM}$, vastus intermedius (VI), rectus femoris (RF), sartorius (SAR), gracilis, adductor longus, adductor magnus, biceps femoris short and heads, semintendinosus, semimembranosus) were manually delimited using FSLView (FMRIB, Oxford, USA). Muscles were delineated in every two slices. Corresponding masks on the remaining slices were generated using an interpolated process (Ogier et al. 2017). Using the truncated cone formula, muscle volume of each muscle was calculated by summing areas of all the slices, taking into account the slice thickness and the gaps between slices.

$T_{2}$ maps were generated using a linear fit on a pixel-bypixel basis using the following equation:

$\ln (S(T E))=\ln \left(S_{0}\right)-\left(T E / T_{2}\right)$,

where $\mathrm{S}(\mathrm{TE})$ is the signal at time equal to echo time and $\mathrm{S}_{0}$ is the equilibrium magnetization. Regions of interest initially drawn on $T_{1}$-weighted images were used to analyse $T_{2}$ maps and to determine a mean $T_{2}$ value for each muscle.

The reproducibility of measurements (e.g., MVC force, volume, $T_{2}$ ) was reported in previous studies (Fouré et al. $2014,2015 \mathrm{a})$. The number of participants was determined on the basis of a statistical power calculation $(\alpha=0.05$ and $1-\beta=0.9$ ) and previous measurements (Fouré et al. 2014) with the aim of detecting a significant increase of $7 \%$ in the mean $T_{2}$ of the whole quadriceps femoris. Normality of the data distribution was initially investigated using Shapiro-Wilk test. Two-way repeated-measure ANOVA (time $\times$ muscle length) was used (Statistica, Statsoft, Tulsa, USA) to assess changes in parameters assessed during the NMES session (i.e., stimulation intensity and relative output force), MVC force, soreness, volume and $T_{2}$ in each muscle. Sphericity hypothesis was checked with a Mauchly's test and results were adjusted using the Greenhouse-Geisser correction if required. A Tukey's HSD post hoc analysis was used when appropriate. The level of significance was set at $P<0.05$ and results are reported as mean \pm standard deviation (SD).

\section{Results}

During the NMES session, stimulation intensity was increased similarly for both muscle length conditions and reached $59 \pm 10 \mathrm{~mA}$ (Fig. 2a). The resulting force level was significantly larger for the short muscle length as compared to the long muscle length condition $(P<0.01$, Fig. 2b). The mean relative force output recorded throughout the NMES sessions was $23 \pm 7 \%$ MVC force at long muscle length and $45 \pm 15 \%$ MVC force at short muscle length. The maximal electrically-evoked force reached $30 \pm 5 \%$ MVC force and $61 \pm 12 \%$ MVC force when NMES was performed at long and short muscle length, respectively.

The relative MVC force was significantly lower for the long length at D2, D4 and D7 compared to the short length $(P<0.01)$. For the long muscle length condition, MVC force decreased by $-28 \pm 7 \%$ immediately after the NMES session (from PRE: $408 \pm 110 \mathrm{~N}$ to POST: $301 \pm 85 \mathrm{~N}$ ) and was still lower than the baseline value at D2 $(-34 \pm 10 \%$, $276 \pm 85 \mathrm{~N}), \mathrm{D} 4(-27 \pm 7 \%, 303 \pm 89 \mathrm{~N})$ and D7 $(-21 \pm 5 \%$, $326 \pm 93 \mathrm{~N}$ ) (Fig. 2c). On the contrary, for the short muscle length condition, MVC force remained unchanged immediately after, at D2 and at D4 but significantly increased at D7 $(+17 \pm 13 \%$, from PRE: $401 \pm 148 \mathrm{~N}$ to $\mathrm{D} 7: 453 \pm 143 \mathrm{~N}$, $P<0.001)$.

The amount of soreness at D2 was significantly higher at long length compared to the short length condition $(P<0.01)$. Muscle soreness significantly increased and reached its maximum at D2 for the long muscle length condition (30 $\pm 24 \mathrm{~mm}$, Fig. $2 \mathrm{~d})$. No significant change in soreness was detected for the short muscle length condition $(P>0.05)$.

Muscle volume and $T_{2}$ were increased in muscles of both limbs after the NMES session (Table 1). A larger increase was found for the long muscle length condition (Fig. 3) especially for the two muscles located beneath the stimulation electrodes (i.e., VL and VM).

Regarding muscle volume, a statistical time effect was found at both short and long muscle lengths for SAR at D2, D4 and D7 $(P<0.05$, Table 1$)$. A significant time $\times$ muscle length interaction was found for VL and VM $(P<0.01$ and $P<0.05$, respectively) likely due to a significant muscle volume increase at long muscle length for VM (D2: $+5 \pm 5 \%$, D $4:+5 \pm 5 \%$ and $\mathrm{D} 7:+3 \pm 4 \%)$ and VL (D2: $+8 \pm 4 \%$, D4: $+12 \pm 7 \%$ and D7: $+12 \pm 9 \%$ ).

A significant time effect was also found for $T_{2}$ changes at both short and long muscle length for SAR at D2, D4 and D7 $(P<0.01$, Table 1$)$. Additionally, time $\times$ muscle length interactions were identified for $\mathrm{T}_{2}$ in the four muscles of the quadriceps femoris $(P<0.01)$. Overall, a significant $T_{2}$ increase was observed at long muscle length 


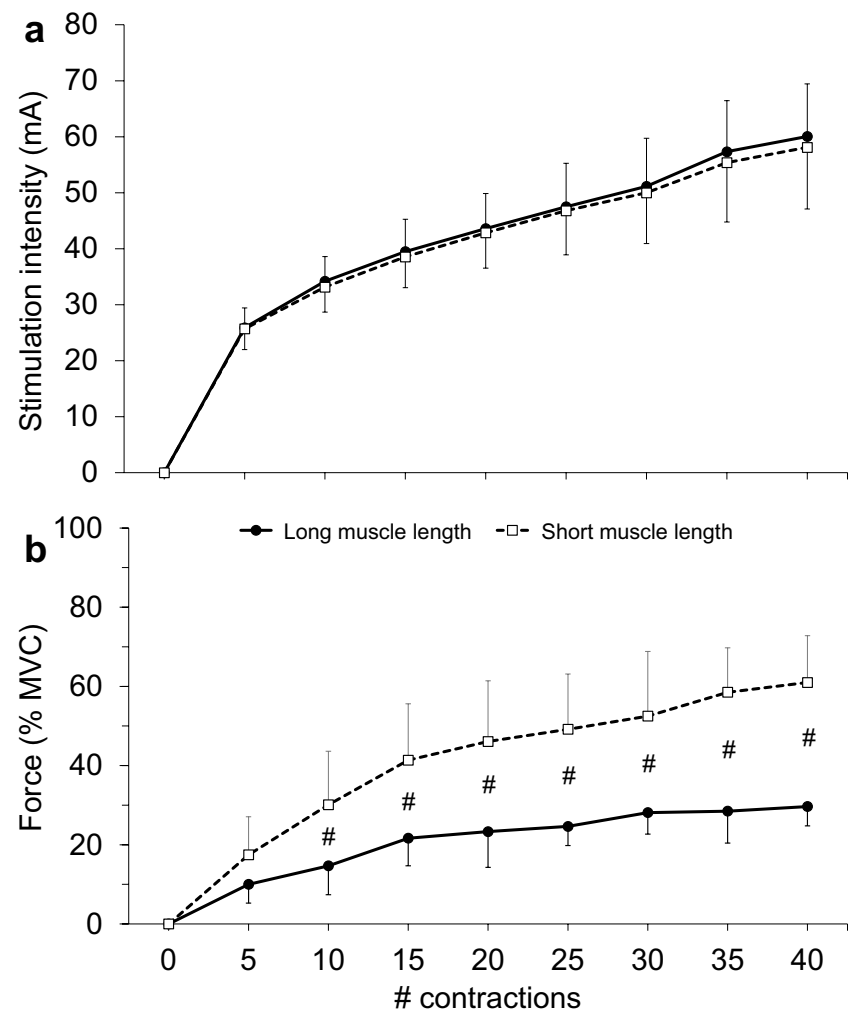

Fig. 2 a Time course of current stimulation intensity and $\mathbf{b}$ relative force production during the neuromuscular electrical stimulation (NMES) session performed with knee flexed at $100^{\circ}$ (solid line: long muscle length) and knee flexed at $50^{\circ}$ (dotted line: short muscle length). c Maximal voluntary contraction (MVC) force and d muscle soreness assessed from visual analog scale (VAS) score before (PRE), immediately after and 2, 4 and 7 days (POST, D2, D4 and D7, respectively) after the neuromuscular electrical stimulation (NMES) session

for RF (D2: $+5 \pm 5 \%, \mathrm{D} 4:+6 \pm 6 \%$ and D7: $+5 \pm 5 \%$ ), VI (D2: $+3 \pm 3 \%, \mathrm{D} 4:+4 \pm 4 \%$ and D7: $+3 \pm 3 \%)$, VM (D2: $+6 \pm 4 \%, \mathrm{D} 4:+11 \pm 7 \%$ and $\mathrm{D} 7:+11 \pm 7 \%)$ and $\mathrm{VL}$ (D2: $+14 \pm 6 \%$, D4: $+27 \pm 14 \%$ and D7: $+36 \pm 18 \%)$. In addition, a significant increase from D2 to D4 was observed for VM $(+4 \pm 4 \%)$ and $\mathrm{VL}(+12 \pm 9 \%)$. For the other thigh muscles (i.e., not involved in knee extension), experimental conditions mostly had no effect on muscle volume and $T_{2}$ changes (see Supplementary Table).

\section{Discussion}

The main results of the present study demonstrated that the occurrence and extent of isometric NMES-induced muscle damage in knee extensors are lower at short muscle length. Long-lasting MVC force reduction and large muscle $T_{2}$ increase were recorded in quadriceps femoris muscles within days following the NMES session performed at long
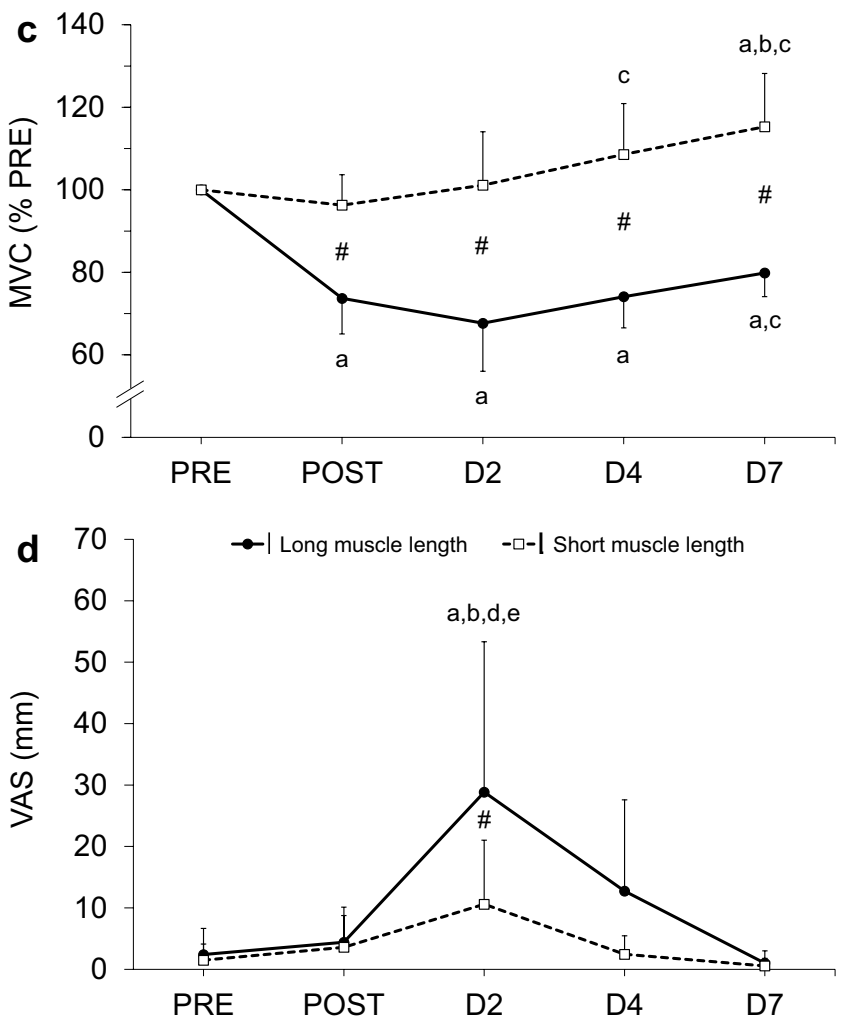

performed with knee flexed at $100^{\circ}$ (solid line: long muscle length) and knee flexed at $50^{\circ}$ (dotted line: short muscle length). Error bars represent standard deviations. a: significantly different from PRE $(P<0.05)$, b: significantly different from POST $(P<0.05)$, c: significantly different from D2 $(P<0.05)$, d: significantly different from D4 $(P<0.05)$, e: significantly different from D7 $(P<0.05)$, \#: significant difference between long and short muscle length conditions $(P<0.05)$

muscle length. These deleterious changes were not observed at short muscle length.

While the stimulation intensity was similar throughout the NMES sessions performed at both short and long muscle length, significantly lower force levels were produced by the knee extensors at long muscle length as compared to the short length condition. This reduced force production under similar stimulation conditions might be due to a lower involvement of both VL and VM muscles. A less-efficient force production by knee extensor muscles resulting from the force-length relationship might also been advocated. Accordingly, based on force-length relationships assessed in human cadavers, Herzog et al. demonstrated that the individual force produced by the vastii muscles was maximal for a knee angle near $50^{\circ}-80^{\circ}$ in isometric conditions (Herzog et al. 1990). It is noteworthy that the peak torque measured in vivo during knee extensors isometric MVC occurs around $70^{\circ}$ of knee flexion as reported previously (Knapik et al. 1983; Marginson and Eston 2001; Lanza et al. 2019). 


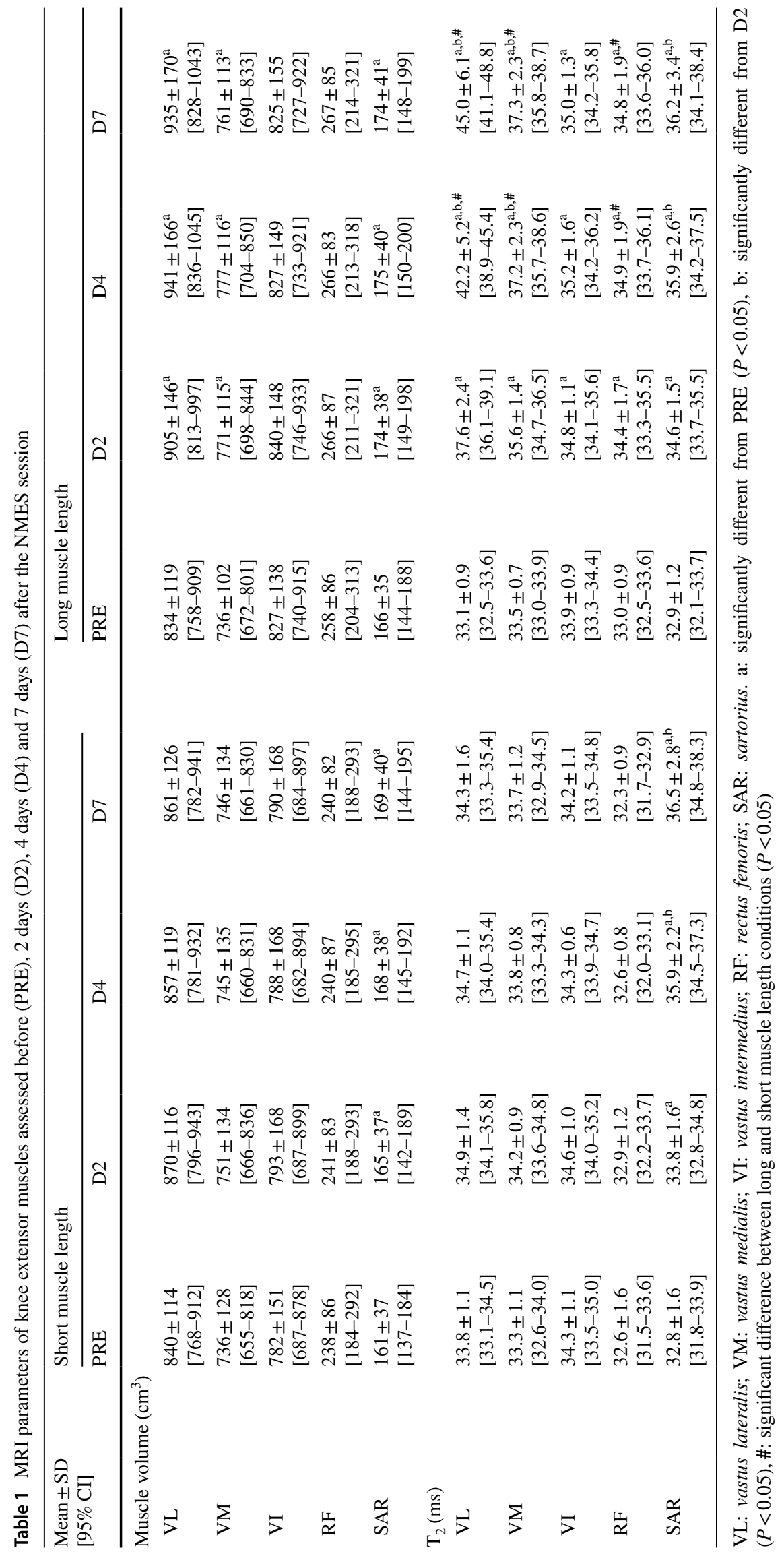


Fig. 3 Axial $T_{2}$ maps superimposed on $T_{1}$-weighted images (exposed in the upper part with muscle delineations for $A D D$ $L$ : adductor longus, ADD-M: adductor magnus, BF-S: biceps femoris short head, BF-L: biceps femoris long head, GR: gracilis, RF: rectus femoris,

SAR: sartorius, SM: semimembranosus, ST: semintendinosus, VI: vastus intermedius, VM: vastus medialis, VL: vastus lateralis) acquired on a representative participant with a comparison of the explorations performed before (PRE), 2 days (D2), 4 days (D4) and 7 days (D7) after the isometric NMES session at long and short muscle length during the NMES exercise. Changes in $T_{2}$ maps colour are due to muscle alterations resulting from the damaging exercise
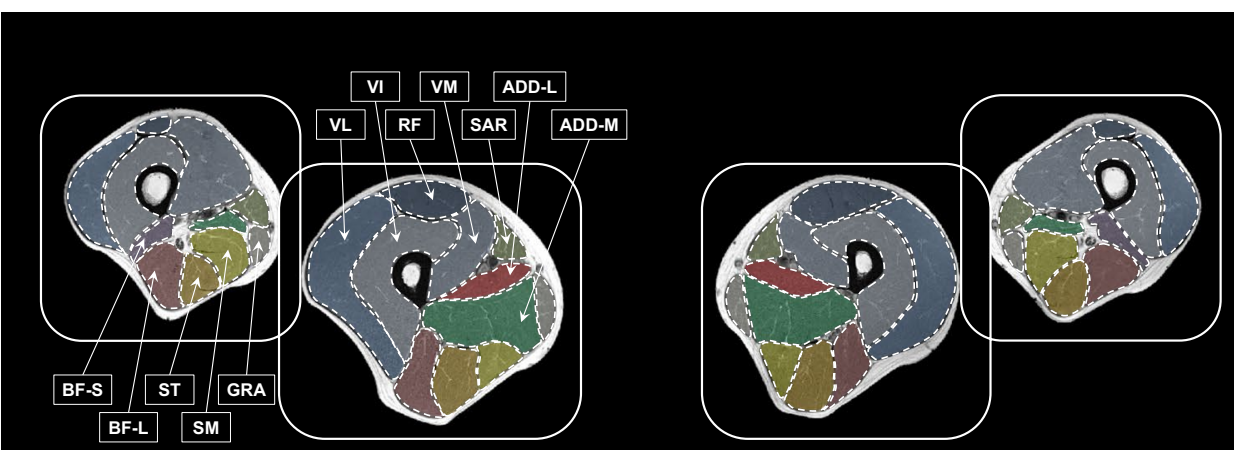

$\mathrm{T}_{2}$ (ms)
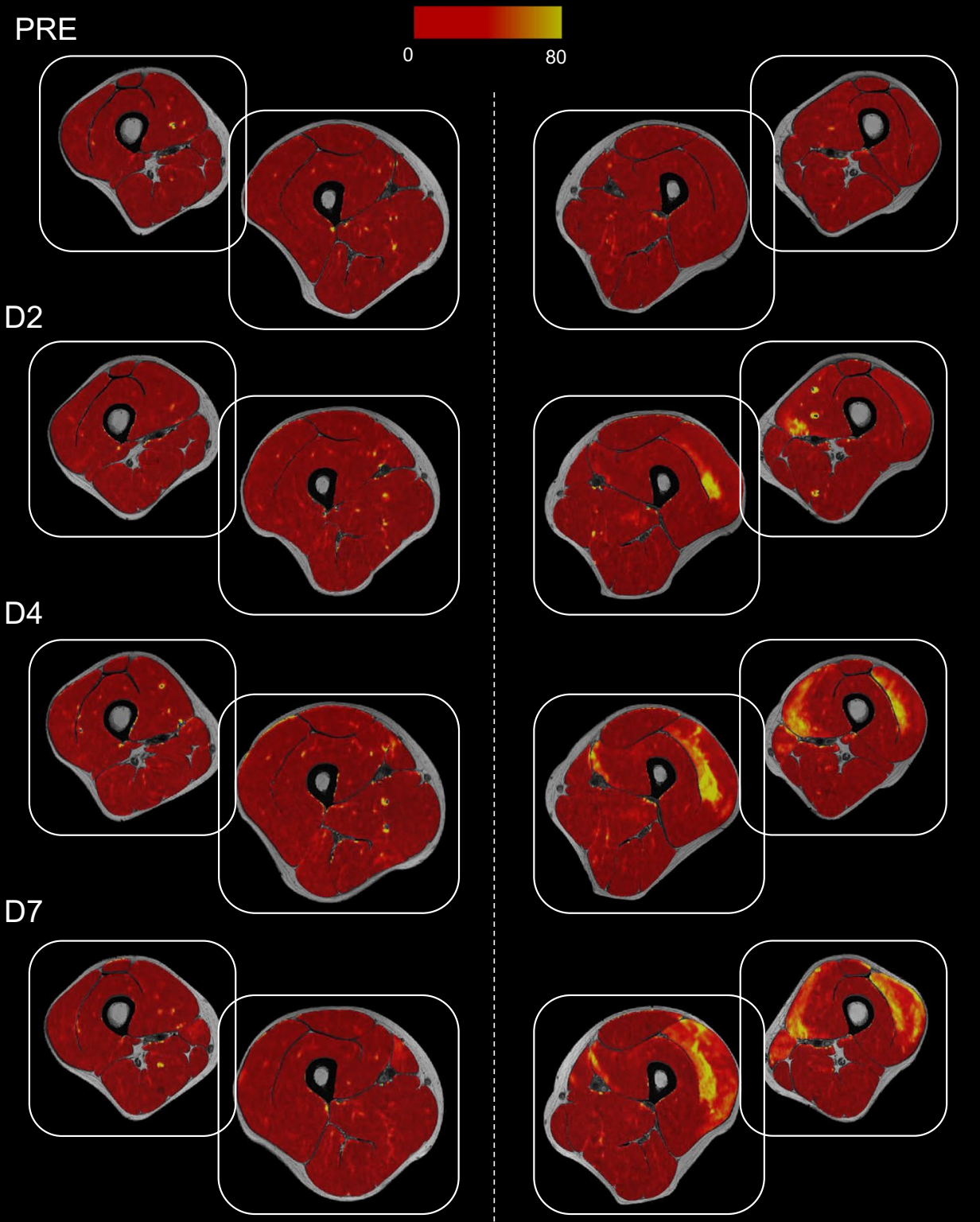

Short muscle length

\section{Long muscle length}


Surprisingly and despite the twofold lower electricallyevoked force resulting from the NMES session performed at long muscle length, larger muscle alterations were recorded as illustrated by the large MVC force reduction, the increased muscle soreness and $T_{2}$ when compared to the short length condition. MVC force dropped after the long muscle length NMES session with a time-course similar to what has been previously described (Aldayel et al. 2010; Nosaka et al. 2011; Fouré et al. 2014). An additional longlasting effect was measured at D7 $(-21 \pm 5 \%)$ illustrating an incomplete MVC force recovery resulting from the severe muscle damage (Paulsen et al. 2012). In contrast, a reduced MVC force or an increased muscle soreness was recorded neither immediately after nor within days following the short muscle length NMES session. Similarly, muscle volume and $T_{2}$ were significantly increased in the directly stimulated muscles during the long muscle length condition, whereas no change occurred for the short muscle length condition. These significant differences illustrated a muscle length dependence of NMES-induced damage in agreement with what has been recently reported $24 \mathrm{~h}$ after maximal voluntary isometric contractions (Allen et al. 2018). The musclelength dependence of isometric NMES-induced damage could be related, at least in part, to the so-called popping sarcomere hypothesis (Morgan and Proske 2004). Accordingly, considering the sarcomere length-tension curve (Morgan 1990), Allen et al. (2018) suggested that the overstretching of some sarcomeres beyond the myofilaments overlap can account for the initiation of muscle damage. In addition, both muscle fascicle lengthening and muscle tension were involved in the amount of muscle damage following eccentric contractions (Guilhem et al. 2016). On that basis, muscles similarly stimulated at a long muscle length would be more sensitive to damage than those activated at a short muscle length. Considering that the motor unit recruitment associated with NMES is mainly superficial (Maffiuletti 2010), the localization of $T_{2}$ changes in the superficial part of the VM muscle within days after the exercise performed at long muscle length would support the assumption of active sarcomeres overstretch. However, in the VL, sarcomeres length might change a little over the range of knee angles used in the present study (Herbert et al. 2002). A mean sarcomere length change of about $0.4 \mu \mathrm{m}$, representing an increase of about $10 \%$ between the two knee flexion positions, has been recently measured in the VL using microendoscopy (Chen et al. 2016). Although the mean sarcomere length change was relatively minor, the tension capacity may have been altered. In addition, between the two knee flexion positions used in the present study, the length change of VL and VM muscle-tendon units has been of about $6-7 \%$ on the basis of measures performed on cadavers (Visser et al. 1990). Therefore, the resistance to the shortening sarcomeres during contraction could have been higher due to a potential shorter "toe region" of the passive structures in series within the muscle-tendon unit. However, a recent study demonstrated that muscle alterations were mainly located in less activated areas following the NMES exercise (Fouré et al. 2019). Therefore, the deeper localization of $T_{2}$ changes for the VL muscle would suggest that other mechanisms than the popping sarcomere hypothesis are involved.

The increased muscle $T_{2}$ in the deep part of the VL muscle might be associated with the alteration of costameres and/or passive components of muscle tissue. From a comparative analysis between muscle damage induced by voluntary and NMES eccentric contractions, Crameri et al. (2007) indicated that a significant disruption of cytoskeletal proteins (i.e., desmin) only occurred after the NMES contractions. In addition, ultrasound elastography measurements have recently illustrated that connective tissues can also be exposed to stretch at long muscle length (Freitas et al. 2019; Xu et al. 2018) contributing to increase the tension on contractile tissues and overstretch during contractions. The increased passive stress and strain on knee extensor muscles during passive stretches can be considered as facilitating conditions for a potential disruption of passive structures including connective tissues. Overall, intramuscular alterations illustrated in the present study by $\mathrm{T}_{2}$ changes could result from damage of the muscle extracellular matrix (Mackey et al. 2008, 2011) and/or structural elements of muscle cells (Crameri et al. 2007; Mackey et al. 2008). Further investigations are warranted to determine the potential alteration of the latter structures in the deep and superficial parts of the VL and VM muscles in response to NMESinduced isometric muscle contractions at long length. One can also put forth that the inhomogeneous activation of the four muscles within the quadriceps femoris group might have disturbed the mechanical coupling between musculotendinous structures (Maas and Finni 2018). The relative displacement of two neighbourhood muscles (e.g., VL and VI) during the isometric NMES protocol could have created a stress between muscle fascias thereby contributing to an increased shear strain on intramuscular connective tissues and so more particularly at long muscle length. Additional investigations would be of interest to assess the behaviour of muscle fibres during the NMES-induced isometric contractions in both knee flexion positions and the potential subsequent changes in local mechanical properties using ultrasound imaging and/or elastography. That would help to understand the biomechanical conditions leading to damage occurring only at long muscle length. We did not measure fascicle length changes during the NMES exercise and this could be viewed as a limitation of the present study. It would have been of interest to assess the strain on active fascicles but also on passive structures such as series elastic components during the NMES-isometric contractions throughout the exercise. The impact of the fascicle/passive structures 
strain at short and long muscle lengths on the extent of muscle $T_{2}$ increase and MVC force decrease could have been assessed as reported recently during voluntary eccentric contractions (Guilhem et al. 2016; Doguet et al. 2019).

Slight changes in muscle volume and $T_{2}$ were also detected for SAR and antagonist muscles independently of the length condition. This might be linked to potential contractions occurring as a result of reflexes, co-activation and/or to preserve knee and hip joints integrity during the NMES sessions. A significant $T_{2}$ increase was also measured in the RF muscle at D4 and D7 for the long muscle length condition. One can hypothesize that water diffusion could have occurred from the neighbourhood muscles (i.e., from VL and VM for which muscle volume increased due to the edema). Overall, our results further support that isometric NMES-induced contractions performed at long muscle length led to severe muscle alterations (Paulsen et al. 2012) as illustrated by large MVC force changes. In addition, the same NMES protocol performed at short muscle length prevented these deleterious changes and was linked to a paradoxical MVC force increase at D7 which would need to be further documented. The contribution of neuromuscular adaptations (e.g., increased intra- and inter-muscular coordination and/or a decreased contribution of knee flexors during knee extension MVC) to a single NMES-isometric contractions session leading to an increase in MVC force 7 days post-exercise observed in the present study cannot be ruled out.

During the NMES session in the present study, a high frequency stimulation protocol with a manual increase in stimulation intensity was used in order to induce muscle damage as described in previous studies (Fouré et al. 2014, 2015a). Similar stimulation intensities were used at both short and long muscle lengths, but a larger relative evoked force was recorded at short muscle length. That phenomenon could be, at least in part, related to a larger muscle volume involvement, a potential difference in VL and VM relative contribution to maximal isometric knee extension and/or a slight shift of stimulation electrodes location on the muscles already described as the soft tissue artefact (i.e., a skin sliding over the muscle with joint angle change). Regarding the latter potential issue, methodological cautions were taken to accurately stick the electrodes on the muscle belly of each muscle and so for each experimental position. Further investigations based on $T_{2}$ measurements to assess muscle activation and relative contribution at short and long muscle lengths could have been of interest. In addition, it could have been relevant to assess the MVC force length relationship considering that the peak of this relationship can be shifted following a damaging exercise leading to a potential change in MVC force assessed at a single knee angle (Gregory et al. 2007). Therefore, additional assessment of the MVC force-length relationship for the two experimental conditions reported in the present study are needed to clarify the potential influence of the shift of the optimal length for MVC following isometric NMES-induced muscle damage.

Moreover, neuromuscular alterations can occur following NMES-isometric contractions at long muscle length thereby inducing a decreased MVC force and muscle damage as reported previously (Fouré et al. 2014). In the latter study, a disturbance of excitation-contraction coupling was characterized from a prolonged low-frequency electrically-evoked force depression. This result is in accordance with the alteration of neuromuscular transmission after eccentric exerciseinduced muscle damage (Hedayatpour and Falla 2012) and the disturbance in post-synaptic regulation of acetylcholine as a result of neuromuscular junction remodeling at the injured sites (Warren et al. 1999). In addition, a decreased voluntary activation has been reported (Fouré et al. 2014) which can be related to the corticospinal contribution in the decreased MVC force observed following damaging eccentric contractions (Doguet et al. 2019). Based on the $T_{2}$ changes reported in the present study, muscle alterations were highlighted at long muscle length in comparison to short muscle length condition. However, the contribution of neuromuscular adaptations after NMES-isometric contractions inducing muscle damage to the decreased MVC force at long muscle length should also be considered.

Considering that the electrically-evoked force levels remain the main determinant of NMES efficiency (Maffiuletti 2010) and that muscle hypertrophy can occur independently of the occurrence of muscle damage (Damas et al. 2018), we suggest that rehabilitation training programs including electricallyinduced isometric contractions should be performed at short muscle length.

\section{Conclusion}

A muscle length dependence was clearly illustrated regarding damage resulting from NMES-induced isometric muscle contractions. The short muscle length condition was associated to a higher force production during the NMES protocol as compared to the long length protocol, whereas low muscle damage was only found in SAR. The use of NMES at short muscle length minimize the occurrence and extent of muscle damage (as compared to the longer one) and could contribute to the muscle remodelling despite the lack of muscle tissues alterations. Therefore, NMES performed at short muscle length appears of utmost interest in the field of rehabilitation medicine for both deconditioned patients and injured athletes.

Acknowledgements This study was supported by the Centre National de la Recherche Scientifique (CNRS UMR 7339) and the Assistance Publique des Hôpitaux de Marseille (APHM). The authors thank all the subjects who participated in the present study. 
Author contributions $\mathrm{AF}$ and JG conceived and designed research. AF conducted experiments. AF and ACO analysed the data. AF, JG, DB wrote the manuscript. All authors read and approved the manuscript.

\section{Compliance with ethical standards}

Conflict of interest No conflicts of interest, financial or otherwise, are declared by the authors.

\section{References}

Aldayel A, Jubeau M, McGuigan MR, Nosaka K (2010) Less indication of muscle damage in the second than initial electrical muscle stimulation bout consisting of isometric contractions of the knee extensors. Eur J Appl Physiol 108(4):709-717. https://doi. org/10.1007/s00421-009-1278-0

Allen TJ, Jones T, Tsay A, Morgan DL, Proske U (2018) Muscle damage produced by isometric contractions in human elbow flexors. $\mathrm{J}$ Appl Physiol 124(2):388-399. https://doi.org/10.1152/japplphysi ol.00535.2017

Armstrong RB (1984) Mechanisms of exercise-induced delayed onset muscular soreness: a brief review. Med Sci Sports Exerc 16(6):529-538

Brockett CL, Morgan DL, Gregory JE, Proske U (2002) Damage to different motor units from active lengthening of the medial gastrocnemius muscle of the cat. J Appl Physiol 92(3):1104-1110. https://doi.org/10.1152/japplphysiol.00479.2001

Chen X, Sanchez GN, Schnitzer MJ, Delp SL (2016) Changes in sarcomere lengths of the human vastus lateralis muscle with knee flexion measured using in vivo microendoscopy. J Biomech 49(13):2989-2994. https://doi.org/10.1016/j.jbiom ech.2016.07.013

Clarkson PM, Nosaka K, Braun B (1992) Muscle function after exercise-induced muscle damage and rapid adaptation. Med Sci Sports Exerc 24(5):512-520

Crameri RM, Aagaard P, Qvortrup K, Langberg H, Olesen J, Kjaer M (2007) Myofibre damage in human skeletal muscle: effects of electrical stimulation versus voluntary contraction. J Physiol 583(Pt 1):365-380. https://doi.org/10.1113/jphysiol.2007.128827

Damas F, Libardi CA, Ugrinowitsch C (2018) The development of skeletal muscle hypertrophy through resistance training: the role of muscle damage and muscle protein synthesis. Eur J Appl Physiol 118(3):485-500. https://doi.org/10.1007/s00421-017-3792-9

Doguet V, Nosaka K, Guevel A, Ishimura K, Guilhem G, Jubeau $M$ (2019) Influence of fascicle strain and corticospinal excitability during eccentric contractions on force loss. Exp Physiol 104(10):1532-1543. https://doi.org/10.1113/EP087664

Fouré A, Nosaka K, Wegrzyk J, Duhamel G, Le Troter A, Boudinet H, Mattei JP, Vilmen C, Jubeau M, Bendahan D, Gondin J (2014) Time course of central and peripheral alterations after isometric neuromuscular electrical stimulation-induced muscle damage. PLoS ONE 9(9):e107298. https://doi.org/10.1371/journ al.pone. 0107298

Fouré A, Duhamel G, Wegrzyk J, Boudinet H, Mattei JP, Le Troter A, Bendahan D, Gondin J (2015a) Heterogeneity of muscle damage induced by electrostimulation: a multimodal MRI study. Med Sci Sports Exerc 47(1):166-175. https://doi.org/10.1249/MSS.00000 00000000397

Fouré A, Le Troter A, Guye M, Mattei JP, Bendahan D, Gondin J (2015b) Localization and quantification of intramuscular damage using statistical parametric mapping and skeletal muscle parcellation. Sci Rep 5:18580. https://doi.org/10.1038/srep18580
Fouré A, Le Troter A, Ogier AC, Guye M, Gondin J, Bendahan D (2019) Spatial difference can occur between activated and damaged muscle areas following electrically-induced isometric contractions. J Physiol 597(16):4227-4236. https://doi.org/10.1113/ JP278205

Freitas SR, Antunes A, Salmon P, Mendes B, Firmino T, Cruz-Montecinos C, Cerda M, Vaz JR (2019) Does epimuscular myofascial force transmission occur between the human quadriceps muscles in vivo during passive stretching? J Biomech 83:91-96. https:// doi.org/10.1016/j.jbiomech.2018.11.026

Gregory CM, Bickel CS (2005) Recruitment patterns in human skeletal muscle during electrical stimulation. Phys Ther 85(4):358-364

Gregory JE, Morgan DL, Allen TJ, Proske U (2007) The shift in muscle's length-tension relation after exercise attributed to increased series compliance. Eur J Appl Physiol 99(4):431-441. https://doi. org/10.1007/s00421-006-0363-x

Guilhem G, Doguet V, Hauraix H, Lacourpaille L, Jubeau M, Nordez A, Dorel S (2016) Muscle force loss and soreness subsequent to maximal eccentric contractions depend on the amount of fascicle strain in vivo. Acta Physiol (Oxf) 217(2):152-163. https://doi. org/10.1111/apha.12654

Hedayatpour N, Falla D (2012) Non-uniform muscle adaptations to eccentric exercise and the implications for training and sport. J Electromyogr Kinesiol 22(3):329-333. https://doi.org/10.1016/j. jelekin.2011.11.010

Herbert RD, Moseley AM, Butler JE, Gandevia SC (2002) Change in length of relaxed muscle fascicles and tendons with knee and ankle movement in humans. J Physiol 539(Pt 2):637-645. https ://doi.org/10.1113/jphysiol.2001.012756

Herzog W, Abrahamse SK, ter Keurs HE (1990) Theoretical determination of force-length relations of intact human skeletal muscles using the cross-bridge model. Pflugers Arch 416(1-2):113-119

Howell JN, Chleboun G, Conatser R (1993) Muscle stiffness, strength loss, swelling and soreness following exercise-induced injury in humans. J Physiol 464:183-196

Jamurtas AZ, Theocharis V, Tofas T, Tsiokanos A, Yfanti C, Paschalis V, Koutedakis Y, Nosaka K (2005) Comparison between leg and arm eccentric exercises of the same relative intensity on indices of muscle damage. Eur J Appl Physiol 95(2-3):179-185. https:// doi.org/10.1007/s00421-005-1345-0

Knapik JJ, Wright JE, Mawdsley RH, Braun J (1983) Isometric, isotonic, and isokinetic torque variations in four muscle groups through a range of joint motion. Phys Ther 63(6):938-947. https ://doi.org/10.1093/ptj/63.6.938

Lacourpaille L, Nordez A, Hug F, Couturier A, Dibie C, Guilhem G (2014) Time-course effect of exercise-induced muscle damage on localized muscle mechanical properties assessed using elastography. Acta Physiol (Oxf) 211(1):135-146. https://doi.org/10.1111/ apha. 12272

Lacourpaille L, Nordez A, Hug F, Doguet V, Andrade R, Guilhem G (2017) Early detection of exercise-induced muscle damage using elastography. Eur J Appl Physiol 117(10):2047-2056. https://doi. org/10.1007/s00421-017-3695-9

Lanza MB, Balshaw TG, Folland JP (2019) Is the joint-angle specificity of isometric resistance training real? And if so, does it have a neural basis? Eur J Appl Physiol. https://doi.org/10.1007/s0042 1-019-04229-z

Lau WY, Blazevich AJ, Newton MJ, Wu SS, Nosaka K (2015) Assessment of muscle pain induced by elbow-flexor eccentric exercise. J Athl Train 50(11):1140-1148. https://doi. org/10.4085/1062-6050-50.11.05

Lieber RL, Friden J (1993) Muscle damage is not a function of muscle force but active muscle strain. J Appl Physiol 74(2):520-526

Maas H, Finni T (2018) Mechanical coupling between muscle-tendon units reduces peak stresses. Exerc Sport Sci Rev 46(1):26-33. https://doi.org/10.1249/JES.0000000000000132 
Mackey AL, Bojsen-Moller J, Qvortrup K, Langberg H, Suetta C, Kalliokoski KK, Kjaer M, Magnusson SP (2008) Evidence of skeletal muscle damage following electrically stimulated isometric muscle contractions in humans. J Appl Physiol 105(5):1620-1627. https ://doi.org/10.1152/japplphysiol.90952.2008

Mackey AL, Brandstetter S, Schjerling P, Bojsen-Moller J, Qvortrup K, Pedersen MM, Doessing S, Kjaer M, Magnusson SP, Langberg $\mathrm{H}$ (2011) Sequenced response of extracellular matrix deadhesion and fibrotic regulators after muscle damage is involved in protection against future injury in human skeletal muscle. FASEB J 25(6):1943-1959. https://doi.org/10.1096/fj.10-176487

Maffiuletti NA (2010) Physiological and methodological considerations for the use of neuromuscular electrical stimulation. Eur J Appl Physiol 110(2):223-234. https://doi.org/10.1007/s0042 1-010-1502-y

Marginson V, Eston R (2001) The relationship between torque and joint angle during knee extension in boys and men. J Sports Sci 19(11):875-880. https://doi.org/10.1080/026404101753113822

Morgan DL (1990) New insights into the behavior of muscle during active lengthening. Biophys J 57(2):209-221. https://doi. org/10.1016/S0006-3495(90)82524-8

Morgan DL, Proske U (2004) Popping sarcomere hypothesis explains stretch-induced muscle damage. Clin Exp Pharmacol Physiol 31(8):541-545. https://doi.org/10.1111/j.1440-1681.2004.04029.x

Nosaka K, Clarkson PM (1996) Changes in indicators of inflammation after eccentric exercise of the elbow flexors. Med Sci Sports Exerc 28(8):953-961

Nosaka K, Newton M, Sacco P, Chapman D, Lavender A (2005) Partial protection against muscle damage by eccentric actions at short muscle lengths. Med Sci Sports Exerc 37(5):746-753

Nosaka K, Aldayel A, Jubeau M, Chen TC (2011) Muscle damage induced by electrical stimulation. Eur J Appl Physiol 111(10):2427-2437. https://doi.org/10.1007/s00421-011-2086-x

Ogier A, Sdika M, Fouré A, Le Troter A, Bendahan D (2017) Individual muscle segmentation in MR Images: a 3D propagation through
2D non-linear registration approaches. Paper presented at the 39th Annual International Conference of the IEEE Engineering in Medicine and Biology Society (EMBC'17), Jeju Island, Korea,

Paulsen G, Mikkelsen UR, Raastad T, Peake JM (2012) Leucocytes, cytokines and satellite cells: what role do they play in muscle damage and regeneration following eccentric exercise? Exerc Immunol Rev 18:42-97

Saka T, Akova B, Yazici Z, Sekir U, Gur H, Ozarda Y (2009) Difference in the magnitude of muscle damage between elbow flexors and knee extensors eccentric exercises. J Sports Sci Med 8(1):107-115

Stubbs PW, Walsh LD, D'Souza A, Heroux ME, Bolsterlee B, Gandevia SC, Herbert RD (2018) History-dependence of muscle slack length following contraction and stretch in the human vastus lateralis. J Physiol 596(11):2121-2129. https://doi.org/10.1113/ JP275527

Visser JJ, Hoogkamer JE, Bobbert MF, Huijing PA (1990) Length and moment arm of human leg muscles as a function of knee and hipjoint angles. Eur J Appl Physiol Occup Physiol 61(5-6):453-460

Warren GL, Ingalls CP, Shah SJ, Armstrong RB (1999) Uncoupling of in vivo torque production from EMG in mouse muscles injured by eccentric contractions. J Physiol 515(Pt 2):609-619. https://doi. org/10.1111/j.1469-7793.1999.609ac.x

Xu J, Hug F, Fu SN (2018) Stiffness of individual quadriceps muscle assessed using ultrasound shear wave elastography during passive stretching. J Sport Health Sci 7(2):245-249. https://doi. org/10.1016/j.jshs.2016.07.001 